Proceedings of the 43rd "Jaszowiec", International School and Conference on the Physics of Semiconductors, Wisła 2014

\title{
Optical Properties of SbSI Photonic Crystals
}

\author{
M. Kępińska*, A. Starczewska, P. Duka, M. Nowak and P. SzPerlich \\ Silesian University of Technology, Institute of Physics - Center for Science and Education, \\ Bolesława Krzywoustego 2, 44-100 Gliwice, Poland
}

\begin{abstract}
This paper presents optical properties of $\mathrm{SiO}_{2}$ opals infiltrated with SbSI and inverted SbSI opals for the first time. Registered reflectance spectra exhibit Bragg's peaks connected with photonic band gap. Calculated photonic band structure has been compared with experimental results.
\end{abstract}

DOI: $10.12693 /$ APhysPolA.126.1115

PACS: 78.67.Pt, 77.84.Lf, 42.70.Qs, 42.25.Gy

\section{Introduction}

In recent years, a tremendous amount of research effort has been devoted to preparation of photonic crystals because of the great potential applications in advanced optical devices such as super prisms, waveguides, optical filters and switches, microcavity, and ultimately optical integrated circuits [1].

Antimony sulfoiodide (SbSI) being a semiconducting [2] ferroelectric [3] seems to be a promising material for production of photonic crystals. This material exhibits a wide range of interesting and potentially useful properties. Among them there are the pyroelectric, pyrooptic, piezoeletric, and electrooptic effects (see e.g. [4, 5]). It can be also used as gas sensor $[4,6,7]$.

The aim of this work was to investigate for the first time optical properties of opals filled with SbSI (direct opals) and inverted opals obtained after removing $\mathrm{SiO}_{2}$ templates from SbSI matrixes.

\section{Experiment}

The monodisperse silicon $\left(\mathrm{SiO}_{2}\right)$ nanospheres have been synthesized and gravity sedimented to obtain threedimensional opal templates with a closed-packed face centered cubic (fcc) lattice. These opals have been infiltrated with melted SbSI to produce SbSI direct opals and etched in HF acid to produce SbSI inverted opals. The details of production of the investigated photonic crystals have been presented elsewhere [8 9]. Optical properties of the fabricated structures have been investigated by reflectance spectroscopy in room temperature for wavelengths $(\lambda)$ from $28 \mathrm{~nm}$ to $100 \mathrm{~nm}$. Investigations have been performed using PC2000 (Ocean Optics Inc.) spectrophotometer with master card equipped with appropriate reflection probe $\mathrm{R} 7 \times 400-2-\mathrm{LOH}$ and the deuterium-halogen light source DH2000-FHS from Ocean Optics Inc. The illuminated opals have been mounted

* corresponding author; e-mail: miroslawa.kepinska@polsl.pl horizontally, perpendicular to the light beam. For angular investigations samples have been mounted on the table of GUR-5 (LOMO) goniometer. The measurements have been performed in unpolarized light. The multiple averaged spectral characteristics of optical reflectance $(R)$ containing 2048 data points for various wavelengths have been registered using the OOI-Base program from Ocean Optics Inc

\section{Results and discussion}

Typical spectra $R(\lambda)$ registered for bare $\mathrm{SiO}_{2}$ opals and SbSI direct opals as well as SbSI inverted opals is presented in Fig. 1. One can identify the main Bragg diffraction peaks (connected with photonic pseudogap between 2nd and 3rd band) on spectra registered for $\mathrm{SiO}_{2}$ bare opal and SbSI inverted opal. For SbSI direct opal the main Bragg diffraction peak is invisible in the investigated spectral range. The large red-shift of the Bragg peak is due to the change of effective refractive index of investigated material.

The Bragg peaks positions are shifted to shorter wavelengths with the increase of the angle of light incidence $(\theta)$ (Fig. 2). The angular dependences of Bragg's peaks positions for SbSI direct opal and SbSI inverted opal have been fitted with the modified form of Bragg's law

$$
\lambda_{\mathrm{c}}=2 d \sqrt{n_{\mathrm{eff}}^{2}-\sin ^{2} \theta},
$$

where $d=\sqrt{2 / 3} D$ is the interplanar spacing between (111) planes in photonic crystal, $D$ is the diameter of silica spheres, $n_{\mathrm{eff}}^{2}=n_{\mathrm{sph}}^{2} f_{\mathrm{sph}}+n_{\mathrm{SbSI}}^{2}\left(1-f_{\mathrm{sph}}\right)$ is the effective refractive index, $f_{\mathrm{sph}}$ is the filling factor of photonic crystal with the spheres (for ideal fcc structure $f_{\text {sph }}$ equals 0.74$), n_{\mathrm{sph}}$ and $n_{\mathrm{SbSI}}$ are the refractive indices of silica spheres and surrounding SbSI medium, respectively. According to [10], the refractive index of $\mathrm{SiO}_{2}$ spheres equals $n_{\mathrm{sph}}=1.425$.

In first step $D$ and $n_{\text {eff }}$ have been determined from the data for SbSI direct opal (Table). Assuming that the voids between $\mathrm{SiO}_{2}$ spheres have been completely filled with SbSI and the opal template has ideal fcc structure, refractive index of SbSI $n_{\mathrm{SbSI}}=3.11(1)$ has been estimated. Next, fitting of $\lambda_{c}(\theta)$ for SbSI inverted opal has been done assuming that $D$ has not been changed. 


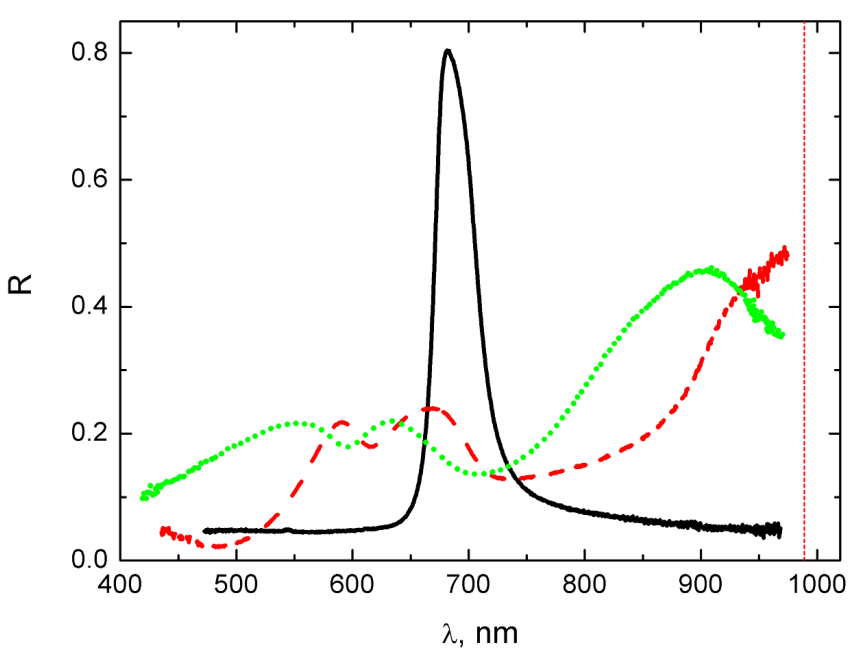

Fig. 1. Spectra of optical reflectance measured for bare opal ( - ) , SbSI direct opal ( - - - ) and SbSI inverted opal obtained after removing $\mathrm{SiO}_{2}$ templates from the SbSI matrixes by 1 min. etching in $10 \% \operatorname{HF}(\ldots$.$) );$ the vertical line shows the position of Bragg peak determined from fitting of angular dependence presented in Fig. 2 for SbSI direct opal.

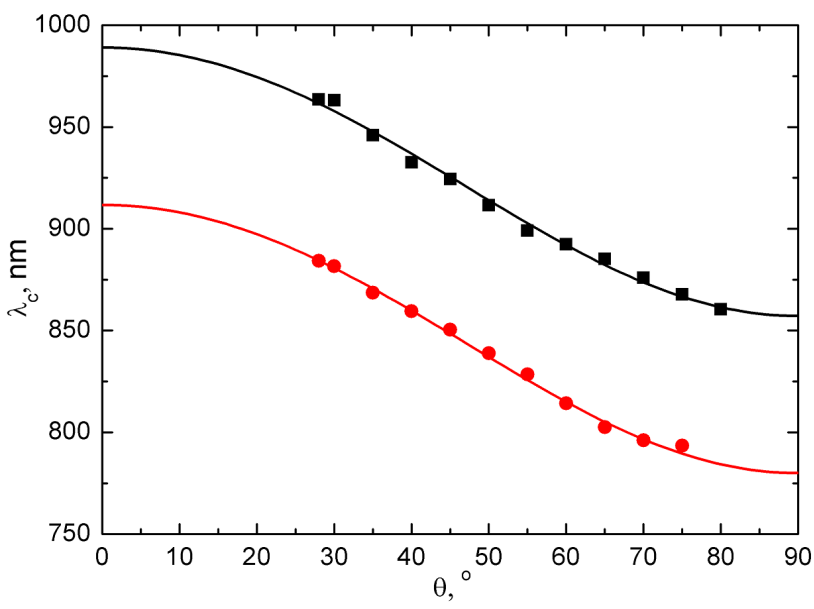

Fig. 2. Angular dependences of the spectral positions of diffraction peaks in reflectance spectra of SbSI direct

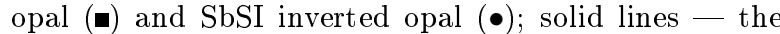
least square fitted dependence (1). Values of the fitted parameters are given in Table.

TABLE

Parameters of SbSI direct and inverted opals evaluated by least squares fitting of the angular dependences of the spectral positions of diffraction peaks in reflectance spectra (Fig. 2).

\begin{tabular}{c|c|c|c|c|c}
\hline \hline & $\lambda_{\mathrm{c}}[\mathrm{nm}]$ & $\lambda_{\mathrm{c}}[\mathrm{nm}]$ & $D[\mathrm{~nm}]$ & $n_{\mathrm{eff}}$ & $n_{\mathrm{SbSI}}$ \\
\cline { 2 - 6 } & $R\left(\theta=0^{\circ}\right)$ & \multicolumn{4}{|c}{$R(\theta)$} \\
\hline $\begin{array}{c}\text { SbSI } \\
\text { direct opal }\end{array}$ & - & $989(2)$ & $302(4)$ & $2.00(2)$ & $3.11(1)$ \\
\hline $\begin{array}{c}\text { SbSI } \\
\text { inverted opal }\end{array}$ & $905(2)$ & $912(2)$ & 302 & $1.863(2)$ & -
\end{tabular}

Values of the fitted parameters are given in Table. One can see that $n_{\text {eff }}$ has decreased after etching. It is connected with the change of refractive index of spheres from refractive index of $\mathrm{SiO}_{2}\left(n_{\mathrm{SiO} 2}\right)$ to refractive index of air $\left(n_{\text {air }}\right)$. Based on the value of $n_{\text {SbSI }}$ determined for SbSI direct opal, $\mathrm{n}_{\mathrm{eff}}$ for inverted opal should be equal to 1.804 in the case of complete etching of $\mathrm{SiO}_{2}$ spheres in the sample. The value of $n_{\text {eff }}$ obtained for investigated SbSI inverted opal is greater. This indicates that after $1 \mathrm{~min}$ etching the $\mathrm{SiO}_{2}$ spheres have not been completely removed from the sample. Therefore, the spheres remaining in SbSI inverted opals after etching may consist in part of $\mathrm{SiO}_{2}$ and in part of air. In this case, similar to the case presented in [11], the expression describing effective refractive index of composite opal structures could take the form

$$
\left.n_{\mathrm{eff}}^{2}-n_{\mathrm{SiO} 2}^{2} f_{\mathrm{sph}}-f_{\mathrm{air}}\right)+n_{\mathrm{air}}^{2} f_{\mathrm{air}}+n_{\mathrm{SbSI}}^{2}\left(1-f_{\mathrm{sph}}\right),
$$

where $f_{\text {air }}$ is the filling factor of photonic crystal with air, which replaces the glass after etching it. This formula may be used to determine $f_{\text {air }}$, and thus to monitor the etching process if other parameters i.e. $n_{\mathrm{eff}}, n_{\mathrm{SiO} 2}$ $n_{\text {air }}, n_{\mathrm{SbSI}}, f_{\mathrm{sph}}$ are known. The filling factor of photonic crystal with air has been calculated using formula

$$
f_{\mathrm{air}}=\frac{n_{\mathrm{SbSI}}^{2}\left(1-f_{\mathrm{sph}}\right)+n_{\mathrm{SiO} 2}^{2} f_{\mathrm{sph}}-n_{\mathrm{eff}}^{2}}{n_{\mathrm{SiO} 2}^{2}-1} .
$$

Assuming that $n_{\mathrm{SbSI}}=3.11$ and $n_{\mathrm{eff}}=1.863$ (see Table), $n_{\mathrm{SiO} 2}=1.425[10]$ and $f_{\mathrm{sph}}=0.74$, the value of $f_{\text {air }}$ equals 0.53 . Etching degree calculated as the percentage ratio of $f_{\text {air }}$ to $f_{\text {sph }}$ is equal to $71.6 \%$.

In order to understand the optical properties of investigated materials, the photonic bands structures have been calculated using plane wave expansion method (MPB software) [12]. It allows to calculate spectral positions and widths of the bandgaps of infinite structures. Only the first 11 bands are plotted in Fig. 3. Comparison of the theoretical results with reflectance spectra measured for normal incidence of light on (111) surface of $\mathrm{SiO}_{2}$ bare opal, SbSI direct opal and SbSI inverted opal are presented in Fig. 3, too.

\section{Conclusions}

Optical reflectance spectra for SbSI direct and inverted photonic crystals have been reported for the first time. Effective refractive indices can be determined using angular dependences of the Bragg peak spectral positions. Applying this method refractive index $n_{\text {SbSI }}=3.11(1)$ of SbSI filling the $\mathrm{SiO}_{2}$ template in SbSI direct opal has been determined. It is proposed to estimate the etching efficiency of $\mathrm{SiO}_{2}$ template by using angular dependences of the Bragg peak spectral positions for SbSI inverted opals. Photonic band structures calculated for SbSI direct opal and SbSI inverted opal are rather well compared with experimental data. The full photonic gap between 8th and 9th is observed in the case of SbSI inverted opal. 


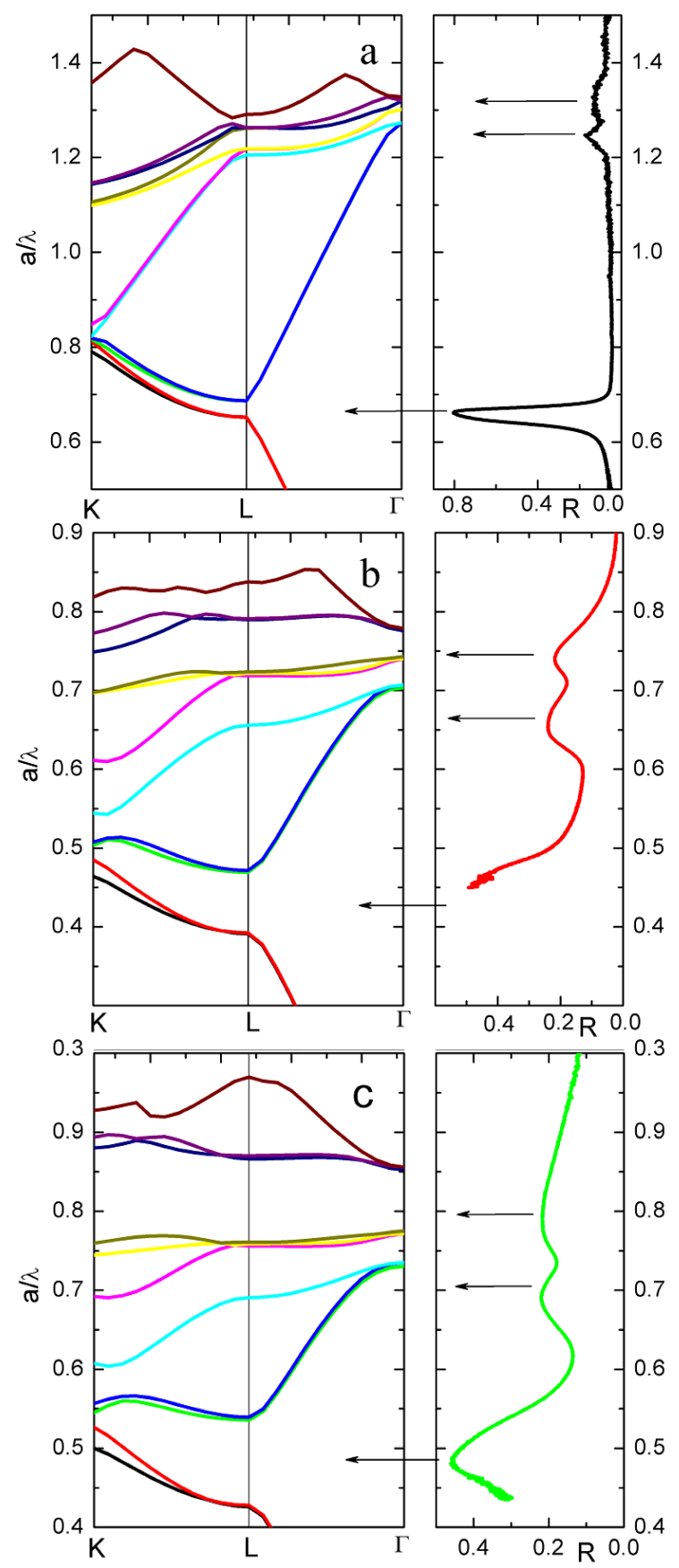

Fig. 3. Photonic bands calculated for $\mathrm{SiO}_{2}$ bare opal (a), SbSI direct opal (b) and SbSI inverted opal (c) compared with measured reflectance spectra.

\section{Acknowledgments}

This work was partially supported by the NCN (Poland) under Contract No. NN507250140.

\section{References}

[1] S.-Y. Hsiao, D.S.H. Wong, S.-Y. Lu, J. Am. Ceram. Soc. 88, 974 (2005).

[2] M. Nowak, P. Szperlich, Opt. Mater. 35, 1200 (2013).

[3] P. Szperlich, M. Nowak, Ł. Bober, J. Szala, D. Stróż, Ultrason. Sonochem. 16, 398 (2009).

[4] E.I. Gerzanich, V.A. Lyakhovitskaya, V.M. Fridkin, B.A. Popovkin, in: SbSI and Other Ferroelectric $A^{\mathrm{V}} B^{\mathrm{VI}} C^{\mathrm{VII}}$ Materials, Ed. E. Kaldis, Current Topics in Materials Science, North-Holland, Amsterdam 1982 , p. 55.

[5] H. Dittrich, N. Karl, S. Kück, W. Schock, in: Ternary Compounds Organic Semiconductors, Ed. O. Madelung, Landolt-Börnstein Condensed Matter III/41E, Springer, Berlin 2000.

[6] M. Nowak, A. Nowrot, P. Szperlich, M. Jesionek, M. Kępińska, A. Starczewska, K. Mistewicz, D. Stróż, J. Szala, T. Rzychoń, E. Talik, R. Wrzalik, Sens. Actuat. A 210, 119 (2014).

[7] A. Starczewska, M. Nowak, P. Szperlich, B. Toron, K. Mistewicz, D. Stróż, J. Szala, Sens. Actuat. A 183, 34 (2012).

[8] M. Kępińska, A. Starczewska, J. Szala, Opt. Mater. 36, 932 (2014).

[9] A. Starczewska, P. Szperlich, M. Nowak, I. Bednarczyk, J. Bodzenta, J. Szala, Acta Phys. Pol. A 126 , 1118 (2014).

[10] A. Chiappini, C. Armellini, A. Chiasera, M. Ferrari, Y. Jestin, M. Mattarelli, M. Montagna, E. Moser, G. Nunzi Conti, S. Pelli, G.C. Righini, M. Clara Goncalves, Rui M. Almeida, J. Non-Cryst. Solids 353, 674 (2007).

[11] M. Kepińska, A. Starczewska, I. Bednarczyk, J. Szala, P. Szperlich, K. Mistewicz, Mater. Lett. 130, 17 (2014).

[12] S. Johnson, J. Joannopoulos, Opt. Express 8, 173 (2001). 